\title{
Can we reduce the recurrence of cornual pregnancy? A case report
}

\author{
Radwan Faraj • Martin Steel
}

Received: 31 January 2008 / Accepted: 26 February 2008 / Published online: 4 April 2008

(C) Springer-Verlag 2008

\begin{abstract}
In this paper, we discuss a successful attempt to reduce the incidence of recurrent cornual pregnancy by ipsilateral tubal occlusion in a lady with two previous cornual pregnancies and a healthy looking contralateral tube.
\end{abstract}

Keywords Cornual pregnancy · Interstitial pregnancy · Tubal occlusion · Cornuostomy

\section{Case report}

A 33-year-old nulliparous woman presented to the Early Pregnancy Fetal Assessment Unit at 6 weeks gestation according to her menstrual dates with vaginal spotting for a week. She had previous history of right-sided cornual pregnancy eight months ago, predicted by empty uterus on transvaginal scan and abnormal serum hCG rise. This was 1,400, 2,100 and 3,000 IU/L at 48-h intervals. Diagnostic laparoscopy confirmed the diagnosis of unruptured right cornual pregnancy. At that time, medical management was offered and the patient received a single dose of systemic methotrexate (MTX). Her serum hCG levels declined after

Note: Patient consent was sought and given for the publication of her case.

\section{R. Faraj $(\bowtie)$}

Department of Obstetrics and Gynaecology,

Royal Bolton Hospital,

Minerva Road,

Farnworth BL4 0JR, UK

e-mail: faraj68@hotmail.com

M. Steel

Blackpool Victoria Hospital,

Blackpool, UK a small initial rise. It took four weeks to reach negative hCG levels.

In this current pregnancy, she had a positive urine pregnancy test at home. A transvaginal scan showed an empty uterus with endometrial thickness at $7 \mathrm{~mm}$, with no fluid in the pelvis. Serum hCG was 5,700 IU/L and the patient was counselled regarding the risk of ectopic pregnancy, but she declined intervention at that time. Serum hCG serially performed at 48-h intervals were 7,800 and $9,700 \mathrm{IU} / \mathrm{L}$, respectively. At the day of the last hCG, the transvaginal scan now showed an adnexal mass of dimensions $2.7 \times 2.2 \times 1.8 \mathrm{~cm}$ in the right cornua, with an endometrial thickness of $13 \mathrm{~mm}$.

In view of this unsatisfactory rise in serum $\mathrm{hCG}$ and scan result, a diagnostic laparoscopy was performed. The operative findings were as follows:a right cornual swelling of dimensions $2 \times 2 \mathrm{~cm}$; the right tube and both ovaries looked normal; the left tube was normal in length with a beaded appearance. At that stage, suction evacuation of the uterus was performed in view of possible failing intrauterine pregnancy (consent was already given for this), which revealed minimal tissues.

Histology of uterine curettage showed fragments of deciduas with arias stella reaction, no trophoblastic tissue and no foetal or placental tissue.

In view of these findings, a medical management of ectopic pregnancy was counselled. The patient received the first dose of methotrexate. The serum hCG peaked to 20,000 IU/L after 5 days of commencing the treatment. A repeat injection of methotrexate was administered to the patient. The serum hCG values declined slowly and took 10 weeks for the level to be undetectable.

In view of this repeated cornual pregnancy and a finding of an abnormal mass in the cornua, the patient was advised to have a hysterosalpingogram before contemplating another 
pregnancy. This was reported as follows:the contrast filling of the uterine cavity was satisfactory, with free spillage of the dye from the left tube; on the right side, there was a circumscribed accumulation of contrast in the region of the right cornua and there was no spillage of the dye.

The tube had an appearance of hydrosalpinx secondary to peritubal adhesions (Fig. 1).

A lengthy discussion and counselling was commenced with the patient and her partner. She was advised to have a right tubal occlusion in an attempt to reduce the chance of repeat cornual pregnancy on this side. Laparoscopic unilateral Filshie clip application (Femcare) to the right fallopian tube was performed. At this time, the abnormal shape of the right cornua was noted, which looked like a small cornual fibroid.

Ten months later, the patient fell pregnant, and is currently in at her 20 weeks of gestation without any problems.

\section{Discussion}

Despite its rarity, cornual (interstitial) pregnancy poses a significant diagnostic and therapeutic challenge, and carries a greater maternal mortality risk than ampullary ectopic pregnancies. In general, the rate of deaths from ectopic pregnancies has not declined since the last Confidential Enquiry into Maternal and Child Health (CEMACH) report, and is still an increase on the rates described for 19911993. There were ten deaths from ruptured ectopic pregnancies: eight in the extrauterine tube and one in the interstitial portion of the tube (cornual pregnancies). In the previous report, four deaths were related to cornual pregnancy. Cornual pregnancies account for $2-4 \%$ of ectopic pregnancies and are said to have a mortality rate in the range $2.0-2.5 \%$ [1].

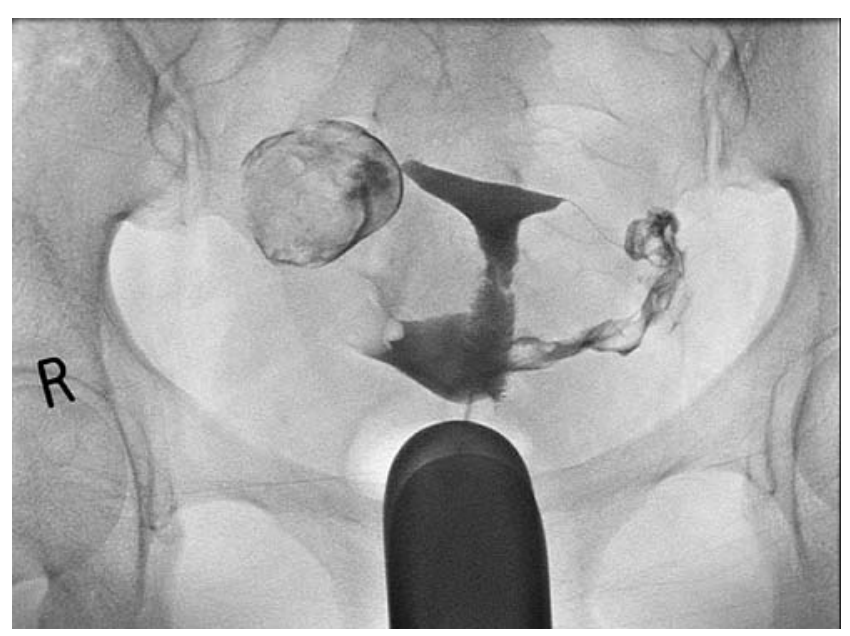

Fig. 1 Hysterosalpingography image showing the patient's left tube and circumscribed contrast accumulation on the left side
Because of myometrial distensibility, cornual pregnancies tend to present relatively late, at 7 to 12 weeks gestation. Significant maternal haemorrhage leading to hypovolaemia and shock can rapidly result from cornual rupture.

Clinically, risk factors are as for other types of ectopic pregnancy. Namely, ipsilateral salpingectomy, previous ectopic pregnancy and in vitro fertilisation are predisposing factors for interstitial pregnancy [2].

The diagnosis can be made with transabdominal or transvaginal ultrasound, using three criteria: (1) empty uterus, (2) a gestational sac seen separately and $<1 \mathrm{~cm}$ from the most lateral edge of the uterine cavity and (3) a thin myometrial layer surrounding the sac [3].

Traditionally, the treatment of cornual pregnancy has been hysterectomy or cornual resection by laparotomy. As all surgical management has been associated with morbidity and unfavourable effects on fertility, more conservative approaches have been introduced into clinical practice. Medical treatment (as with other types of tubal pregnancy) has been introduced with generally satisfactory results [4].

In general, laparoscopic techniques involve cornual resection, cornuostomy, salpingostomy or salpingectomy.

Systemic methotrexate (MTX) is a safe and highly effective treatment for cornual pregnancy. Surgery can be avoided in the majority of women with this condition. Early recognition of the cornual pregnancy with transvaginal ultrasound is essential.. Haemodynamic stability is an essential prerequisite for medical management, and there should be no signs of rupture. The initial level of hCG may predict the need for the second dose MTX, which can be decided depending on follow up hCG trends or clinical symptoms.

Selective uterine artery embolisation has been used after systemic MTX or after the failure of this medical treatment. The authors recommended its use in selected cases of early cornual pregnancy, and it could reduce the haemorrhagic risk [5].

A significant concern after the conservative management of a cornual pregnancy is the recurrence of ectopic pregnancy, namely, cornual pregnancy on the same side. This risk will continue, despite there being a good anatomical result (whether by hysterosalpingography or direct laparoscopic visualisation) following laparoscopic conservative surgery [6].

Tubal pathology is often the primary factor blamed for recurrence. Other factors, such as assisted conception and the non-invasive management of cornual pregnancy, have been shown to contribute to a higher risk of recurrence of cornual pregnancy [7].

Wittich reported an association of recurrent ectopic pregnancy with uterine fibroids [8].

In our case, we think that the abnormally shaped uterine cornu due to a small fibroid is the contributory factor in 
recurrence. Even total salpingectomy will not eliminate the risk of recurrence of ipsilateral cornual pregnancy [9].

The authors propose that, if the chance of transit of the fertilised ovum through the same side of the previous cornual ectopic is eliminated, this may reduce the chance of recurrence. Ipsilateral tubal occlusion, in that case, will be the ideal intervention and we used the Filshie clip application for that purpose. This may not completely prevent recurrence due to zygote transmigration, but will definitely reduce it, as in our case.

Considering the rarity of this type of ectopic pregnancy, there are no randomised controlled trials comparing conservative management with cornuostomy versus salpingectomy or cornual resection. Ipsilateral tubal occlusion in our case may not decrease the rate of subsequent intrauterine pregnancy. If we apply the cohort studies that specifically compare laparoscopic conservative and radical treatments of tubal pregnancy in general, we find that the rate of subsequent intrauterine pregnancy is similar (intrauterine pregnancy $60 \%$ versus $54 \%$, RR $1.11,95 \%$ CI $0.74-1.68$ ), but there was a trend towards higher subsequent ectopic pregnancy in the salpingotomy arm (recurrent ectopic pregnancy $18 \%$ versus $8 \%$, RR $2.38,95 \%$ CI $0.57-10.01$ ) $[10,11]$.

We are aware of the lifetime chance of sterilisation failure of 1:200 possibly due to recanalisation of the tube, and this can happen in our case. However, in the absence of strong evidence, we still believe that ipsilateral tubal occlusion in cases of recurrent cornual pregnancy with a normal-looking contralateral tube is a justified attempt to reduce the risk of recurrent cornual pregnancy.

In general, the available data in the literature are conflicting in guiding the appropriate counselling regarding future pregnancy risks and the optimum mode of delivery following cornual pregnancy.

\section{References}

1. Department of Health (2007) Report on confidential enquiries into maternal deaths in the United Kingdom 2003-2005. HMSO, London

2. Tulandi T, Al-Jaroudi D (2004) Interstitial pregnancy: results generated from the Society of Reproductive Surgeons Registry. Obstet Gynecol 103(1):47-50

3. Timor-Tritsch IE, Monteagudo A, Matera C, Veit CR (1992) Sonographic evolution of cornual pregnancies treated without surgery. Obstet Gynecol 79:1044-1049

4. Faraj R, Steel M (2007) Management of cornual (interstitial) pregnancy. Obstet Gynaecol 9(4):249-255

5. Deruelle P, Lucot JP, Lions C, Robert Y (2005) Management of interstitial pregnancy using selective uterine artery embolization. Obstet Gynecol 106:1165-1167

6. Gleicher N, Karande V, Rabin D, Pratt D (1994) Laparoscopic removal of twin cornual pregnancy after in vitro fertilization. Fertil Steril 61:1161-1162

7. van der Weiden RM, Karsdorp VH (2005) Recurrent cornual pregnancy after heterotopic cornual pregnancy successfully treated with systemic methotrexate. Arch Gynecol Obstet 273 (3):180-181

8. Wittich AC (1998) Recurrent cornual ectopic pregnancy in a patient with leiomyomata uteri. J Am Osteopath Assoc 98(6):332333

9. Agarwal SK, Wisot AL, Garzo G, Meldrum DR (1996) Cornual pregnancies in patients with prior salpingectomy undergoing in vitro fertilization and embryo transfer. Fertil Steril 65(3):659-660

10. Silva PD, Schaper AM, Rooney B (1993) Reproductive outcome after 143 laparoscopic procedures for ectopic pregnancy. Obstet Gynecol 81:710-715

11. Bangsgaard N, Lund CO, Ottesen B, Nilas L (2003) Improved fertility following conservative surgical treatment of ectopic pregnancy. Br J Obstet Gynaecol 110:765-770 